Supporting Information

\title{
Robust Hybrid Hydrophilic Coating on a High-Density Polyethylene Surface with Enhanced Mechanical Property
}

Stanislav Klimchuk, Mingwei Shang, Melvin S Samuel, Junjie Niu*

Department of Materials Science and Engineering, CEAS, University of WisconsinMilwaukee, Milwaukee, WI 53211, USA

*E-mail: niu@uwm.edu

Figures S1-S4 


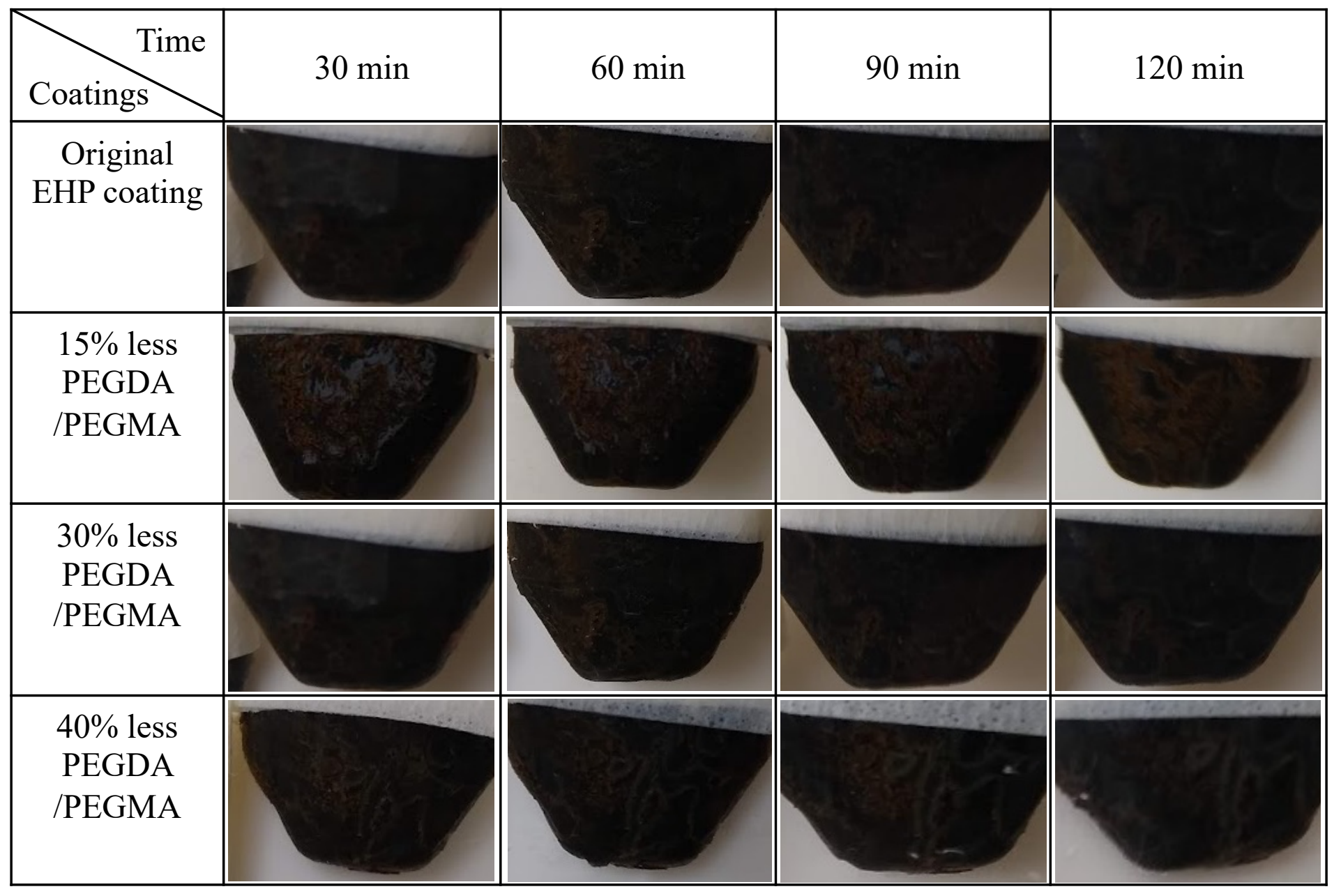

Figure S1. Bubble removal performance of the EHP coated HDPE substrates in DI water under ultrasound agitation with different reducing amounts of PEGDA/PEGMA. The dopamine concentration: $60 \mathrm{mg} / \mathrm{ml}$; epoxy: $850 \mu \mathrm{l}$; temperature: $65^{\circ} \mathrm{C}$. 


\begin{tabular}{|c|c|c|c|c|}
\hline $\begin{array}{c}\text { Time } \\
\text { Dopamine }\end{array}$ & $30 \mathrm{~min}$ & $60 \mathrm{~min}$ & $90 \mathrm{~min}$ & $120 \mathrm{~min}$ \\
\hline $\begin{array}{c}35 \\
\mathrm{mg} / \mathrm{ml}\end{array}$ & & & & \\
\hline $\begin{array}{c}50 \\
\mathrm{mg} / \mathrm{ml}\end{array}$ & & & & \\
\hline $\begin{array}{c}60 \\
\mathrm{mg} / \mathrm{ml}\end{array}$ & & & & \\
\hline $\begin{array}{c} \\
\mathrm{mg} / \mathrm{ml}\end{array}$ & & & & \\
\hline
\end{tabular}

Figure S2. Bubble removal performance of the EHP coated HDPE substrates in DI water under ultrasound agitation with dopamine concentrations of 35, 50, 60 and $70 \mathrm{mg} / \mathrm{ml}$. Epoxy: $850 \mu \mathrm{l}$; PEGDA/PEGMA: $200 \mu \mathrm{L}$; temperature: $65^{\circ} \mathrm{C}$. 


\begin{tabular}{|c|c|c|c|c|}
\hline Temperature & $30 \mathrm{~min}$ & $60 \mathrm{~min}$ & $90 \mathrm{~min}$ & $120 \mathrm{~min}$ \\
\hline $25^{\circ} \mathrm{C}$ & & & & \\
\hline & 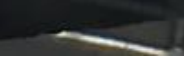 & 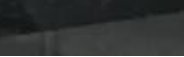 & & 1 \\
\hline $60^{\circ} \mathrm{C}$ & & 3 & & \\
\hline $70^{\circ} \mathrm{C}$ & net & & & \\
\hline $90^{\circ} \mathrm{C}$ & 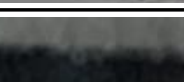 & $a^{2}=$ & & \\
\hline $120^{\circ} \mathrm{C}$ & & & & \\
\hline
\end{tabular}

Figure S3. Bubble removal performance of the EHP coated HDPE subtracts in DI water under ultrasound agitation at different reaction temperatures. Dopamine: 35 mg/ml; epoxy: $850 \mu 1$; PEGDA/PEGMA: $200 \mu \mathrm{L}$. 


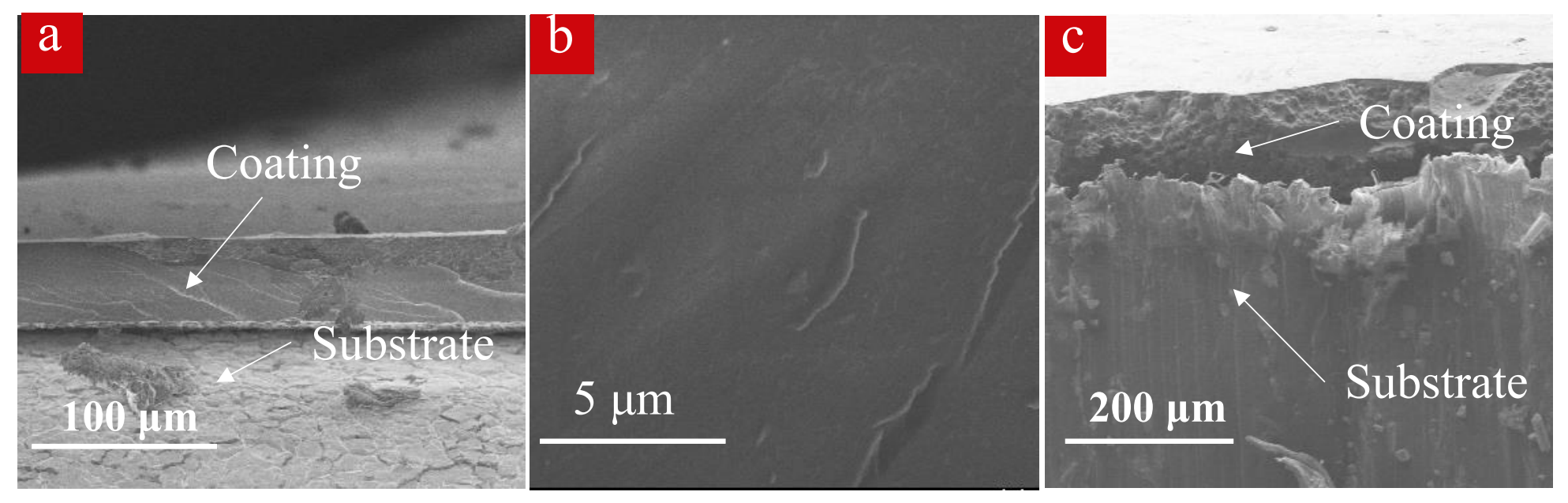

Figure S4. (a) Cross-section and (b) top views of the EHP coating on a steel reflector surface. (c) Cross-section view of the EHP coating on a HDPE surface. 